\title{
OGLE-2015-BLG-0479LA,B: BINARY GRAVITATIONAL MICROLENS CHARACTERIZED BY SIMULTANEOUS GROUND-BASED AND SPACE-BASED OBSERVATIONS
}

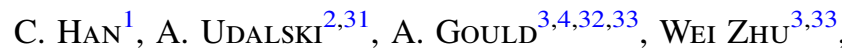

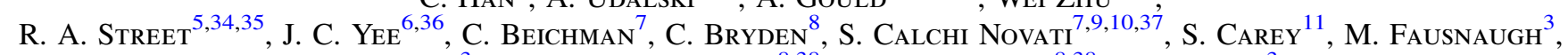 \\ B. S. Gaudi ${ }^{3}$, Calen B. Henderson ${ }^{8,38}$, Y. Shvartzvald ${ }^{8,38}$, B. WibKing $^{3}$
}

(The Spitzer Microlensing Team),

M. K. Szymański ${ }^{2}$, I. Soszyński ${ }^{2}$, J. Skowron ${ }^{2}$, P. Mróz ${ }^{2}$, R. Poleski ${ }^{2,3}$, P. Pietrukowicz ${ }^{2}$, S. KozŁowski ${ }^{2}$, K. UlaczyK $^{2}$, Ł. WYrZYKOWSKI ${ }^{2}$, M. PAWLAK ${ }^{2}$

(The OGLE Collaboration),

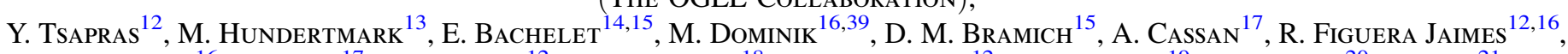
K. Horne ${ }^{16}$, C. Ranc ${ }^{17}$, R. Schmidt ${ }^{12}$, C. Snodgrass ${ }^{18}$, J. Wambsganss ${ }^{12}$, I. A. Steele ${ }^{19}$, J. Menzies ${ }^{20}$, S. MaO ${ }^{21}$

(The RoboNet COLlaboration),

V. Bozza ${ }^{9,10}$, U. G. Jørgensen ${ }^{22}$, K. A. Alsubai ${ }^{15}$, S. Ciceri ${ }^{4}$, G. D’Ago ${ }^{4,9}$, T. Haugbølle ${ }^{22}$, F. V. Hessman ${ }^{23}$, T. C. Hinse ${ }^{24}$,

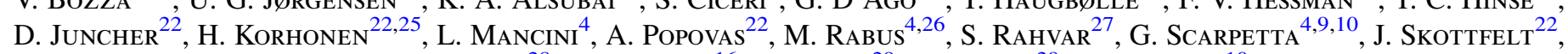
J. Southworth ${ }^{28}$, D. Starkey ${ }^{16}$, J. Surdej ${ }^{29}$, O. Wertz ${ }^{29}$, M. Zarucki ${ }^{10}$

(The MiNDSTEp ConsorTium),

\author{
AND \\ R. W. PogGe ${ }^{3}$, D. L. DePoy ${ }^{30}$ \\ (The $\mu$ FUN Collaboration) \\ ${ }^{1}$ Department of Physics, Chungbuk National University, Cheongju 361-763, Korea \\ ${ }^{2}$ Warsaw University Observatory, Al. Ujazdowskie 4, 00-478 Warszawa, Poland \\ ${ }^{3}$ Department of Astronomy, Ohio State University, 140 W. 18th Avenue, Columbus, OH 43210, USA \\ ${ }^{4}$ Max Planck Institute for Astronomy, Königstuhl 17, D-69117 Heidelberg, Germany \\ ${ }^{5}$ School of Physics and Astronomy, Queen Mary University of London, Mile End Road, London E1 4NS, UK \\ ${ }^{6}$ Harvard-Smithsonian Center for Astrophysics, 60 Garden Street, Cambridge, MA 02138, USA \\ ${ }^{7}$ NASA Exoplanet Science Institute, MS 100-22, California Institute of Technology, Pasadena, CA 91125, USA \\ 8 Jet Propulsion Laboratory, California Institute of Technology, 4800 Oak Grove Drive, Pasadena, CA 91109, USA \\ ${ }_{10}^{9}$ Dipartimento di Fisica "E. R. Caianiello," Uńiversitá di Salerno, Via Giovanni Paolo II, I-84084 Fisciano (SA), Italy \\ ${ }^{10}$ Istituto Internazionale per gli Alti Studi Scientifici (IIASS), Via G. Pellegrino 19, I-84019 Vietri Sul Mare (SA), Italy \\ ${ }^{11}$ Spitzer Science Center, MS 220-6, California Institute of Technology, Pasadena, CA, USA
Astronomisches Rechen-Institut, Zentrum für Astronomie der Universität Heidelberg (ZAH), D-69120 Heidelberg, Germany \\ ${ }^{13}$ Niels Bohr Institute \& Centre for Star and Planet Formation, University of Copenhagen, Øster Voldgade 5, DK-1350-Copenhagen K, Denmark \\ ${ }^{14}$ Las Cumbres Observatory Global Telescope Network, 6740 Cortona Drive, Suite 102, Goleta, CA 93117, USA \\ ${ }^{15}$ Qatar Environment and Energy Research Institute(QEERI), HBKU, Qatar Foundation, Doha, Qatar \\ ${ }^{16}$ SUPA, School of Physics \& Astronomy, University of St. Andrews, North Haugh, St. Andrews KY16 9SS, UK \\ ${ }^{17}$ Sorbonne Universités, UPMC Univ Paris 6 et CNRS, UMR 7095, Institut d'Astrophysique de Paris, 98 bis bd Arago, F-75014 Paris, France \\ ${ }_{18}$ Planetary and Space Sciences, Department of Physical Sciences, The Open University, Milton Keynes, MK7 6AA, UK \\ ${ }_{19}$ Astrophysics Research Institute, Liverpool John Moores University, Liverpool CH41 1LD, UK \\ ${ }^{20}$ South African Astronomical Observatory, P.O. Box 9, Observatory 7935, South Africa \\ ${ }^{21}$ National Astronomical Observatories, Chinese Academy of Sciences, 100012 Beijing, China \\ ${ }^{22}$ Niels Bohr Institutet, Københavns Universitet, Juliane Maries Vej 30, DK-2100 København Ø, Denmark \\ ${ }^{23}$ Institut für Astrophysik, Georg-August-Universität Göttingen, Friedrich-Hund-Platz 1, D-37077 Göttingen, Germany \\ ${ }^{24}$ Korea Astronomy and Space Science Institute, 776 Daedeokdae-ro, Yuseong-gu, 305-348 Daejeon, Korea \\ ${ }^{26}$ Instituto de Astrofísica, Facultad de Física, Pontificia Universidad Cat'olica de Chile, Av. Vicuña Mackenna 4860, 7820436 Macul, Santiago, Chile \\ ${ }^{27}$ Department of Physics, Sharif University of Technology, P.O. Box 11155-9161 Tehran, Iran \\ ${ }^{28}$ Astrophysics Group, Keele University, Staffordshire, ST5 5BG, UK \\ ${ }^{29}$ Institut d'Astrophysique et de Géophysique, Université de Liège, B-4000 Liège, Belgium \\ ${ }^{30}$ Department of Physics and Astronomy, Texas A\&M University, College Station, TX 77843-4242, USA \\ Received 2016 April 2; revised 2016 June 29; accepted 2016 June 30; published 2016 August 29
}

ABSTRACT

We present a combined analysis of the observations of the gravitational microlensing event OGLE-2015-BLG0479 taken both from the ground and by the Spitzer Space Telescope. The light curves seen from the ground and

\footnotetext{
31 The OGLE collaboration.

32 The $\mu$ FUN collaboration.

33 The Spitzer Microlensing Team.

${ }^{34}$ The RoboNet collaboration.

35 The MiNDSTEp consortium.

${ }^{36}$ Sagan Fellow.

37 Sagan Visiting Fellow.

38 NASA Postdoctoral Program Fellow.

39 Royal Society University Research Fellow.
} 
from space exhibit a time offset of $\sim 13$ days between the caustic spikes, indicating that the relative lens-source positions seen from the two places are displaced by parallax effects. From modeling the light curves, we measure the space-based microlens parallax. Combined with the angular Einstein radius measured by analyzing the caustic crossings, we determine the mass and distance of the lens. We find that the lens is a binary composed of two G-type stars with masses of $\sim 1.0 M_{\odot}$ and $\sim 0.9 M_{\odot}$ located at a distance of $\sim 3 \mathrm{kpc}$. In addition, we are able to constrain the complete orbital parameters of the lens thanks to the precise measurement of the microlens parallax derived from the joint analysis. In contrast to the binary event OGLE-2014-BLG-1050, which was also observed by Spitzer, we find that the interpretation of OGLE-2015-BLG-0479 does not suffer from the degeneracy between $( \pm, \pm)$ and $( \pm, \mp)$ solutions, confirming that the four-fold parallax degeneracy in single-lens events collapses into the two-fold degeneracy for the general case of binary-lens events. The location of the blend in the colormagnitude diagram is consistent with the lens properties, suggesting that the blend is the lens itself. The blend is bright enough for spectroscopy and thus this possibility can be checked from future follow-up observations.

Key words: binaries: general - gravitational lensing: micro

\section{INTRODUCTION}

Einstein radii of typical Galactic gravitational microlensing events are of the order of astronomical units. Hence, if lensing events are observed from a satellite in a solar orbit, the relative lens-source positions seen from the ground and from the satellite appear to be different, resulting in different light curves. A combined analysis of the light curves observed both from the ground and from the satellite leads to the measurement of the microlens-parallax vector $\pi_{\mathrm{E}}$ (Refsdal 1966; Gould 1994), which is referred to as the "space-based microlens parallax." The measurement of $\pi_{\mathrm{E}}$ is important because it enables one to constrain the mass $M$ and distance $D_{\mathrm{L}}$ to the lensing object by

$$
M=\frac{\theta_{\mathrm{E}}}{\kappa \pi_{\mathrm{E}}} ; \quad D_{\mathrm{L}}=\frac{\mathrm{au}}{\pi_{\mathrm{E}} \theta_{\mathrm{E}}+\pi_{\mathrm{S}}},
$$

where $\theta_{\mathrm{E}}$ is the angular Einstein radius, $\kappa=4 G /\left(c^{2} \mathrm{au}\right)$, $\pi_{\mathrm{S}}=\mathrm{au} / D_{\mathrm{S}}$ is the parallax of the lensed star (source), and $D_{\mathrm{S}}$ is the distance to the source. Microlens parallaxes can be measured from the single platform of Earth that is being accelerated by its orbital motion around the Sun. Although parallaxes of most lenses with known physical parameters were measured in this way, the ground-based measurement of microlens parallaxes, referred to as annual microlens parallaxes, has limited applicability, primarily to the small fraction of long timescale events caused by nearby lenses. Therefore, a space-based microlens parallax provides the only way to routinely measure microlens parallaxes for an important fraction of microlensing events.

In 2014, the 50 year old concept of the space-based microlens-parallax measurement was realized by a microlensing program making use of the Spitzer Space Telescope (Gould et al. 2014), which has a projected separation from the Earth of $\sim 1$ au. The principal goal of the program is determining the Galactic distribution of planets by measuring microlens parallaxes and thereby estimating distances of the individual lenses (Calchi Novati et al. 2015a). From combined observations both from the ground and from the Spitzer telescope conducted in 2014 and 2015 seasons, the masses and distances of two microlensing planets were successfully determined (Udalski et al. 2015b; Street et al. 2016).

Besides planetary microlensing events, other important target lensing events of Spitzer observations are those produced by binary objects, especially caustic-crossing binary-lens events. Caustics in gravitational lensing phenomena refer to the positions on the source plane at which a point source would be infinitely magnified. In reality, source stars have finite sizes and thus lensing magnifications during caustic crossings deviate from those of a point source. Detecting these finitesource effects enables one to measure the angular Einstein radius $\theta_{\mathrm{E}}$, which is the other ingredient needed for the unique determinations of $M$ and $D_{\mathrm{L}}$ (see Equation (1)). The usefulness of Spitzer observations in characterizing binaries was demonstrated by the microlens-parallax measurements for two caustic-crossing binary-lens events (Shvartzvald et al. 2015; Zhu et al. 2015).

In this paper, we present the analysis of the caustic-crossing binary-lens event OGLE-2015-BLG-0479, which was simultaneously observed by ground-based telescopes and the Spitzer Space Telescope in the 2015 season. By measuring both the lens parallax and the angular Einstein radius, we are able to determine the mass and distance to the lens. In addition, we can constrain the complete orbital parameters of the lens thanks to the precisely measured microlens parallax by the Spitzer data. We also investigate modeling degeneracies by comparing the event with OGLE-2014-BLG-1050 (Zhu et al. 2015), which is another caustic-crossing binary-lens event observed by Spitzer with similar photometric precision, cadence, and coverage.

\section{OBSERVATION}

The event OGLE-2015-BLG-0479 occurred on a star located in the Galactic bulge field with coordinates (R.A., decl. $)_{\mathrm{J} 2000}=\left(17^{\circ} 43^{\prime} 40^{\prime \prime} 6,-35^{\mathrm{h}} 30^{\mathrm{m}} 33^{\mathrm{s}}\right.$. 4$)$, which corresponds to the Galactic coordinates $(l, b)=\left(354^{\circ} .18,-3^{\circ} .08\right)$. It was discovered by the Early Warning System (EWS: Udalski et al. 2015a) of the OGLE group on 2015 March 18 $\left(\mathrm{HJD}^{\prime}=\mathrm{HJD}-2450000 \sim 7100\right)$ from survey observations conducted using the $1.3 \mathrm{~m}$ telescope located at Las Campanas Observatory in Chile.

On 2015 May $13\left(\mathrm{HJD}^{\prime} \sim 7155.5\right)$, the event exhibited a sharp rise of the source brightness and the onset of this anomaly was announced to the microlensing community. Such a rise in the light curve is a characteristic feature that occurs when a source star enters a caustic formed by a binary object. In response to the anomaly alert, the $\mu \mathrm{FUN}$ collaboration (Gould et al. 2006) conducted follow-up observations using the $1.0 \mathrm{~m}$ telescope at the Cerro Tololo Inter-American Observatory (CTIO) in Chile. After the sharp rise, the light curve exhibited a "U"-shape brightness variation, which is a characteristic feature when the source moves inside of a binary caustic. Caustics produced by binary lenses are closed curves, and thus a caustic exit was anticipated. On $\mathrm{HJD}^{\prime} \sim 7191$, the source brightness suddenly dropped, indicating that the source 


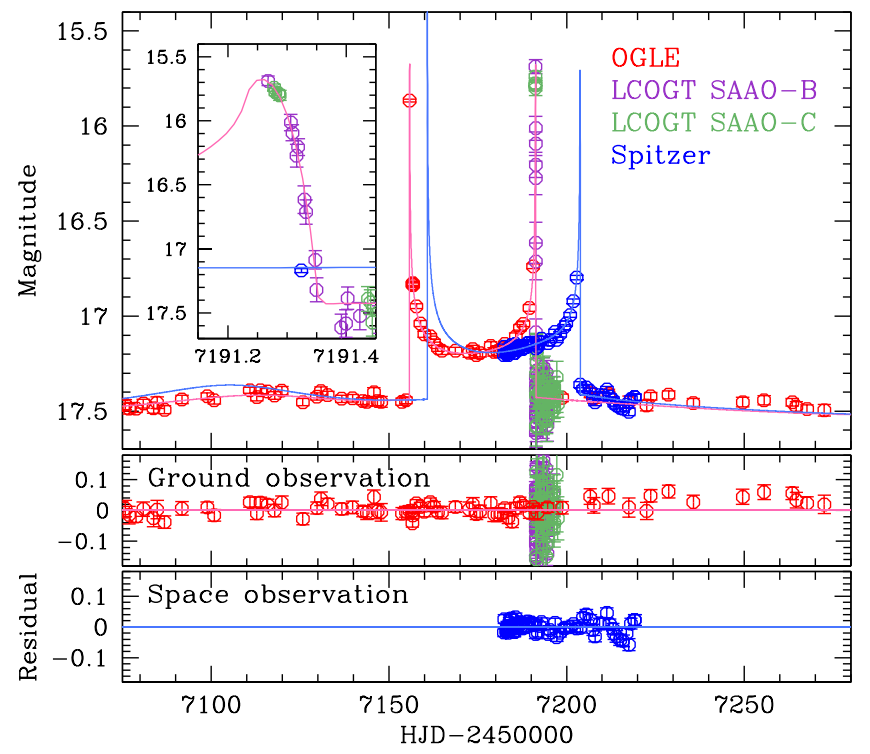

Figure 1. Light curves of the microlensing event OGLE-2015-BLG-0479 as seen from the Earth and from the Spitzer telescope. Superposed on the data points are the best-fit model curves obtained considering space-based parallax effects. The insets show an enlargement of the caustic-exit part of the light curve seen from Earth. The two lower panels show the residuals from the model for the ground-based and space-based data sets.

exited the caustic. The RoboNet collaboration and the MiNDSTEp consortium, who were watching the progress of the event, conducted intensive observations during the caustic exit using two $1.0 \mathrm{~m}$ telescopes of Las Cumbres Observatory Global Telescope Network (LCOGT) located in the South African Astronomical Observatory (SAAO). Thanks to the follow-up observations, the caustic exit was densely resolved.

The event was also observed from space as part of the Spitzer microlensing program. The general description of the program and target selection protocol in 2015 season are given in Udalski et al. (2015b) and Yee et al. (2015), respectively. Spitzer observations were conducted for 37 days from 2015 June $8\left(\mathrm{HJD}^{\prime} \sim 7182\right)$ to July $15\left(\mathrm{HJD}^{\prime} \sim 7219\right)$. The event was observed with a half-day cadence until June 18 (HJD' 7192), just after the caustic exit seen from the ground, and one-day cadence thereafter. From these observations, a total of 59 data points were obtained.

Data from ground-based observations were processed using pipelines that are based on the Difference Image Analysis method (Alard \& Lupton 1998; Woźniak 2000) and customized by the individual groups (Udalski 2003; Bramich 2008). Data from Spitzer observations were processed by using a photometry algorithm that is optimized for images taken by the Infrared Array Camera of Spitzer in crowded fields (Calchi Novati et al. 2015b).

In Figure 1, we present the light curve of OGLE-2015-BLG0479. One finds that both light curves observed from the ground and from the Spitzer telescope are characterized by distinctive caustic-crossing features. We note that both the caustic entrance and exit were captured by the ground-based data, while only the caustic exit was captured by the spacebased data. The light curves observed from the ground and from the Spitzer telescope exhibit an $\sim 13$ day offset between the times of the caustic exits, indicating that the relative lenssource positions are displaced by the parallax effect.
Another important characteristics of the light curves is that the duration between the caustic crossings in the ground-based light curve, 35 days, comprises a significant fraction of the whole duration of the event ( $\sim 180$ days). This indicates that the source is likely to have crossed a big caustic formed by a binary lens with roughly equal mass components and a separation similar to the Einstein radius corresponding to the total mass of the lens. This is further evidenced by the fact that the space-based light curve also exhibits a strong caustic-crossing feature that could not have been produced if the caustic were small compared to the displacement of the source trajectory by parallax effects.

In many respects, OGLE-2015-BLG-0479 is similar to OGLE-2014-BLG-1050 (Zhu et al. 2015), which is another caustic-crossing binary-lens event simultaneously observed from the ground and from the Spitzer telescope. First, the light curves of both events exhibit distinctive caustic-crossing features with wide time gaps between the caustic-crossing spikes. Second, the Spitzer data cover the caustic exit but miss the entrance for both events. Third, both events have similar timescales and were covered with similar photometric precision and cadence. Hence, it will be interesting to compare the results of analysis, particularly regarding the four-fold degeneracy that was identified to exist for OGLE-2014-BLG-1050. See Section 3 for more details about the degeneracy.

\section{MODELING}

Light curves of single-mass lensing events obtained from both space- and ground-based observations yield four sets of degenerate solutions (Refsdal 1966), which are often denoted by $(+,+),(-,-),(+,-)$, and $(-,+)$, where the former and latter signs in each parenthesis represent the signs of the lenssource impact parameters as seen from Earth and from the satellite, respectively. This four-fold degeneracy occurs due to the fact that a pair of light curves resulting from the source trajectories seen from Earth and from the satellite passing on the same side with respect to the lens, i.e., $(+,+)$ or $(-,-)$ solutions, are similar to the pair of light curves resulting from source trajectories passing on the opposite sides of the lens, i.e., $(+,-)$ or $(-,+)$ solutions. For the graphical presentation of the four-fold degeneracy, see Figure 2 of Gould (1994).

For well covered binary-lens events, it is expected that the degeneracy between the pair of $(+,+)$ and $(+,-)$ [or $(-,-)$ and $(-,+)$ ] solutions are generally resolved due to the lack of lensing magnification symmetry compared to the single-lens case. The remaining degeneracy, i.e., $(+,+)$ versus $(-,-)$, may persist, but these solutions usually give similar amplitudes of the microlens parallax, and thus the physical lens parameters estimated from the two degenerate solutions are similar to one another. In the case of OGLE-2014-BLG-1050, Zhu et al. (2015) found that the four-fold degeneracy unexpectedly persisted and diagnosed that the degeneracy remained unresolved because (1) Spitzer data partially covered the light curve and (2) the source-lens relative motion happened to be almost parallel to the direction of the binary-lens axis. Similar to OGLE-2014-BLG-1050, the Spitzer data of OGLE-2015-BLG0479 cover only the caustic exit of the light curve, and thus the degeneracy may persist. We, therefore, investigate the possibility of the degeneracy.

Modeling of the light curve of OGLE-2015-BLG-0479 is carried out in multiple steps:

1. preliminary modeling based on the ground-based data, 
2. measuring the microlens parallax with combined groundand space-based data, and

3. refining the identified solutions.

In the following paragraphs, we describe these in detail.

In the first step, we conduct a preliminary modeling of the light curve obtained from ground-based observations in order to find an initial position in the parameter space from which $\chi^{2}$ minimization can be initiated. This preliminary modeling is based on the seven principal binary lensing parameters plus two flux parameters for the data set obtained by each telescope. The first four of these principal parameters describe the lenssource approach, including $t_{0}, u_{0}, t_{\mathrm{E}}$, and $\alpha$, where $t_{0}$ is the time of the closest source approach to a reference position of the lens, $u_{0}$ is the source-reference separation at $t_{0}$ (impact parameter), $t_{\mathrm{E}}$ is the timescale for the source to cross the angular Einstein radius $\theta_{\mathrm{E}}$ of the lens (Einstein timescale), and $\alpha$ is the angle between the source trajectory and the binary axis (source trajectory angle). We choose the center of mass of the binary lens as the reference position. Another two principal parameters characterize the binary lens including $s_{\perp}$ and $q$, where $s_{\perp}$ is the projected separation and $q$ is the mass ratio between the binary-lens components. We note that the parameters $u_{0}$ and $s_{\perp}$ are normalized to $\theta_{\mathrm{E}}$. The last parameter $\rho$, which is defined as the ratio of the angular source radius $\theta_{*}$ to the Einstein radius, i.e., $\rho=\theta_{*} / \theta_{\mathrm{E}}$ (normalized source radius), is needed to account for the caustic-crossing parts of the light curve affected by finite-source effects. The two flux parameters $F_{s}$ and $F_{b}$ represent the fluxes from the source and blended light, respectively. The principal lensing parameters are searched for by using a downhill approach based on the Markov Chain Monte Carlo (MCMC) method. The flux parameters $F_{s}$ and $F_{b}$ are searched for by a linear fitting.

Magnifications affected by finite-source effects are computed by using a combination of numerical and semi-analytic methods. In the immediate neighboring region around caustics, we use the numerical inverse-ray-shooting method (Schneider \& Weiss 1986). In the outer region surrounding caustics, we use the semi-analytic hexadecapole approximation (Gould 2008; Pejcha \& Heyrovský 2009).

In computing finite-source magnifications, we consider surface brightness variation of the source star caused by limb darkening by modeling the surface brightness profile as

$$
S_{\lambda} \propto\left[1-\Gamma_{\lambda}\left(1-\frac{3}{2} \cos \phi\right)\right],
$$

where $\Gamma_{\lambda}$ is the linear limb-darkening coefficient and $\phi$ is the angle between the normal to the source surface and the line of sight toward the center of the source star. The values of the limb-darkening coefficient are chosen from the catalog of Claret (2000) based on the source type determined from the dereddened color and brightness. We find that the source is an early K-type subgiant and adopt $\Gamma_{I}=0.53$ and $\Gamma_{L}=0.22$. For the detailed procedure of determining the source type, see Section 4.

In the second step, we conduct another modeling including the Spitzer data and considering parallax effects, starting from the solution found from the preliminary modeling. Parallax effects are incorporated by two parameters $\pi_{\mathrm{E}, N}$ and $\pi_{\mathrm{E}, E}$, which are the two components of the lens parallax vector $\pi_{\mathrm{E}}$ projected onto the sky along the north and east equatorial coordinates, respectively. The starting values of the lens parallax parameters
$\pi_{\mathrm{E}, N}$ and $\pi_{\mathrm{E}, E}$ can be, in principle, estimated from the offsets in the values of $t_{0}$ and $u_{0}$ for the two light curves observed from the ground and from the Spitzer telescope because the parallax vector is related to these offsets by

$$
\boldsymbol{\pi}_{\mathrm{E}}=\frac{\mathrm{au}}{D_{\perp}}\left(\frac{\Delta t_{0}}{t_{\mathrm{E}}}, \Delta u_{0}\right),
$$

where $\Delta t_{0}=t_{0, \text { sat }}-t_{0, \oplus}, \Delta u_{0}=u_{0, \text { sat }}-u_{0, \oplus}$, and $D_{\perp}$ is the projected separation between Earth and the satellite. During the time of the event, $D_{\perp} \sim 1.4$ au. However, this analytic estimation of the lens parallax vector is difficult because $t_{0 \text {,sat }}$, and $u_{0 \text {, sat }}$ are uncertain due to the partial coverage of the event by the Spitzer data. Another way to obtain a starting $\pi_{\mathrm{E}}$ value is conducting an additional modeling based on the ground-based data but this time considering the annual parallax effects, which affect the groundbased light curve via Earth's annual orbital motion. We find that implementing this method is also difficult because the photometric data are not good enough and cadence of ground-based observation is not high enough to precisely measure $\pi_{\mathrm{E}}$ based on subtle deviations caused by the annual parallax. We therefore conduct a grid search in the $\pi_{\mathrm{E}, N}-\pi_{\mathrm{E}, E}$ plane. In addition to finding a starting value of $\pi_{\mathrm{E}}$, this second-step grid search is needed to identify possibly multiple solutions resulting from the parallax degeneracy.

In the final step, we identify local solutions found from the second-step grid search and refine them by letting all parameters vary. In this step, we additionally consider the effect of lens-orbital motion, which is known to induce longterm deviations in binary lensing light curves similar to the deviation induced by parallax effects (Park et al. 2013). Orbital effects cause the projected binary separation $s_{\perp}$ and the source trajectory angle $\alpha$ to vary in time. Under the assumption that the orbital period $P$ is much greater than the event timescale, i.e., $P \gg t_{\mathrm{E}}{ }^{40}$, the variations of $s_{\perp}$ and $\alpha$ can be approximated to be linear and the lens-orbital effect is described by two parameters $d s_{\perp} / d t$ and $d \alpha / d t$, which are the linear change rates of the projected binary separation and the source trajectory angle, respectively. For the full consideration of the Kepler orbital motion, on the other hand, one needs two additional parameters $s_{\|}$and $d s_{\|} / d t$, which represent the line-of-sight separation between the binary-lens components and its rate of change, respectively. See Skowron et al. (2011) for the full description of the orbital lensing parameters. In our analysis,

\footnotetext{
${ }^{40}$ A large binary-lens caustic forms when the separation between the binary components is similar to the physical Einstein radius $r_{\mathrm{E}}$, i.e., $a \sim r_{\mathrm{E}}$. The Einstein radius is related to the mass and distance to the lens by

$$
r_{\mathrm{E}} \sim 4 \mathrm{au}\left(\frac{M}{M_{\odot}}\right)\left[\frac{x(1-x)}{0.25}\right]^{1 / 2},
$$

where $\quad x=D_{\mathrm{L}} / D_{\mathrm{S}} \quad$ (Gaudi 2012). With the Kepler law, $(P / \text { year })^{2}=(a / \text { au })^{3} /\left(M / M_{\odot}\right)$, the orbital period is expressed as

$$
P \simeq 8 \text { year }\left(\frac{r_{\mathrm{E}}}{4 \mathrm{au}}\right)^{3 / 2}\left(\frac{M}{M_{\odot}}\right)^{1 / 4}\left[\frac{x(1-x)}{0.25}\right]^{3 / 4} .
$$

Considering that a typical Einstein timescale

$$
t_{\mathrm{E}} \simeq 35 \text { day }\left(\frac{M}{M_{\odot}}\right)^{1 / 2},
$$

the orbital period of a binary lens is much greater than the Einstein timescale, and thus the assumption $P \gg t_{\mathrm{E}}$ is valid in most cases of Galactic binary-lens events.
} 
Table 1

Lensing Parameters

\begin{tabular}{lcc}
\hline \hline Parameters & $u_{0}>0$ & $u_{0}<0$ \\
\hline$\chi^{2}$ & 736.4 & 732.9 \\
$t_{0}(\mathrm{HJD}-2450000)$ & $7163.992 \pm 0.743$ & $7166.439 \pm 0.179$ \\
$u_{0}$ & $0.417 \pm 0.005$ & $-0.418 \pm 0.004$ \\
$t_{\mathrm{E}}($ days $)$ & $91.0 \pm 1.7$ & $86.3 \pm 0.5$ \\
$s_{\perp}$ & $1.07 \pm 0.01$ & $1.10 \pm 0.01$ \\
$q$ & $0.88 \pm 0.05$ & $0.81 \pm 0.03$ \\
$\alpha(\mathrm{rad})$ & $-0.270 \pm 0.017$ & $0.242 \pm 0.003$ \\
$\rho\left(10^{-3}\right)$ & $0.75 \pm 0.14$ & $0.73 \pm 0.12$ \\
$\pi_{\mathrm{E}, N}$ & $0.01 \pm 0.01$ & $-0.06 \pm 0.01$ \\
$\pi_{\mathrm{E}, E}$ & $-0.12 \pm 0.01$ & $-0.11 \pm 0.01$ \\
$d s_{\perp} / d t\left(\mathrm{yr}^{-1}\right)$ & $0.01 \pm 0.08$ & $-0.32 \pm 0.05$ \\
$d \alpha / d t\left(\mathrm{yr}^{-1}\right)$ & $0.53 \pm 0.04$ & $-0.40 \pm 0.01$ \\
$s_{\|}$ & $0.21 \pm 0.28$ & $-1.09 \pm 0.19$ \\
$d s_{\|} / d t\left(\mathrm{yr}^{-1}\right)$ & $0.50 \pm 0.35$ & $-0.01 \pm 0.25$ \\
\hline
\end{tabular}

we test both orbital models based on the linear approximation with two parameters and the full Keplerian orbital motion with four parameters.

\section{SOLUTIONS}

In Table 1, we present the lensing parameters of the solutions found from modeling. We present two sets of solutions with $u_{0}>0$, i.e., $(+,+)$ solution, and $u_{0}<0$, i.e., $(-,-)$ solution, because the degeneracy between the two solutions is very severe with $\Delta \chi^{2} \sim 3.5$. We note that the two degenerate solutions are in mirror symmetry with respect to the binary axis and thus the parameters of the solutions are in the relation $\left(u_{0}, \alpha, \pi_{\mathrm{E}, N}, d \alpha / d t\right) \leftrightarrow-\left(u_{0}, \alpha, \pi_{\mathrm{E}, N}, d \alpha / d t\right)$. The uncertainty of each parameter is determined as the standard deviation of the distribution derived from the MCMC chain. In Figure 1, we present the best-fit model light curve $\left(u_{0}<0\right.$ solution) superposed on the observed data. The model curves for the ground- and space-based data sets are presented in different colors that are in accordance with those of the individual data sets. We find that the model based on the full Keplerian orbital motion provides a better fit than the model based on the linear approximation with $\Delta \chi^{2} \sim 30$.

Figure 2 shows the geometry of the lens system, where the left and right panels are for the $u_{0}>0$ and $u_{0}<0$ solutions, respectively. We note that the degeneracy between the $u_{0}>0$ and $u_{0}<0$ solutions, which is referred to as the "ecliptic degeneracy" (Skowron et al. 2011), is known to exist for general binary-lens events. In each panel, the red and blue curves with arrows represent the source trajectories seen from the ground and from space, respectively, and the closed curve with six cusps represents the caustic. We note that the shape of the caustic varies in time due to the orbital motion of the binary lens. From the geometry, it is found that the sharp spikes were produced by the crossings of the source over the single big caustic formed by a binary having a roughly equal mass ( $q \sim 0.85)$ component with a projected separation similar to the Einstein radius $\left(s_{\perp} \sim 1.1\right)$. The sources seen from the ground and from space took different trajectories where the space-based source trajectory trailed the ground-based trajectory with a time gap of $\sim 13$ days and with a slightly different source trajectory angle. The weak bump at $\mathrm{HJD}^{\prime} \sim 7115$ in the ground-based light curve was produced when the source approached the cusp of the caustic located on the binary axis close to the lower-mass binary component. We find that improvement of the fit with the consideration of the lens-orbital motion is $\Delta \chi^{2} \sim 43.5$. In the Appendix, we discuss the false alarm probabilities (FAPs) associated with the introduction of the additional orbital-motion parameters relative to the standard model.

Although the degeneracy between $u_{0}>0$ and $u_{0}<0$ solutions persists, we find that the degeneracy between $( \pm$, $\pm)$ and $( \pm, \mp)$ solutions is clearly resolved. In Figure 3, we present the lens system geometry and the model light curve corresponding to the $(+,-)$ local solution. We find that although the local solution explains the caustic-crossing features, the fit in the wings of the light curve is poor with $\chi^{2}$ difference from the $(-,-)$ solution $\Delta \chi^{2}=155$. Therefore, the result confirms that the four-fold degeneracy in single-lens events collapses into the two-fold degeneracy in general binary-lens events.

\section{LENS PARAMETERS}

\subsection{Angular Einstein Radius}

In addition to the microlens parallax, one additionally needs to estimate the angular Einstein radius in order to uniquely determine the lens mass and distance. The angular Einstein radius is measured by analyzing the caustic-crossing parts of the light curve that are affected by finite-source effects. This analysis yields the normalized source radius $\rho=\theta_{*} / \theta_{\mathrm{E}}$. By deducing the angular source radius $\theta_{*}$ from the de-reddened color and brightness, the angular Einstein radius is determined by $\theta_{\mathrm{E}}=\theta_{*} / \rho$.

The de-reddened color $(V-\mathrm{I})_{0}$ and brightness $I_{0}$ of the source star are estimated through multiple steps. We first determine the instrumental $I-H$ color based on the $\mu$ FUN CTIO $I$ and $H$-band data by linear regression of fluxes measured at various magnifications during the event. We then convert $I-H$ into $V-I$ using the color-color relation of Bessell \& Brett (1988) and find that $V-I=0.87 \pm 0.04$. The instrumental $I$-band magnitude of the source star, $I=$ 19.6, is estimated based on the $F_{s}$ and $F_{b}$ values determined from modeling of the OGLE data. Once the instrumental color $V-I$ and brightness $I$ are determined, we then calibrate them based on the relative position of the source star in the instrumental color-magnitude diagram with respect to the centroid of the giant clump (GC), for which its de-reddened color and brightness are known to be constant, $(V-\mathrm{I})_{0}$, $\mathrm{GC}=1.06$ (Bensby et al. 2011) and $I_{0, \mathrm{GC}}=14.7$ (Nataf et al. 2013), and thus can be used as a standard candle (Yoo et al. 2004). Figure 4 shows the locations of the source and centroid of GC in the color-magnitude diagram of neighboring stars around the source star. We find that the de-reddened color and brightness of the source star are $(V-I, I)_{0}=(1.04,17.62)$, implying that the source is a K-type subgiant. From these values, we derive $\theta_{*}=1.37 \pm 0.10 \mu$ as by converting $V-I$ into $V-K$ (Bessell \& Brett 1988) and then applying a colorsurface brightness relation (Kervella et al. 2004).

In Table 2, we list the estimated angular Einstein radii for both $u_{0}>0$ and $u_{0}<0$ solutions. Also presented are the geocentric and heliocentric lens-source proper motions. The geocentric proper motion is determined from the measured 

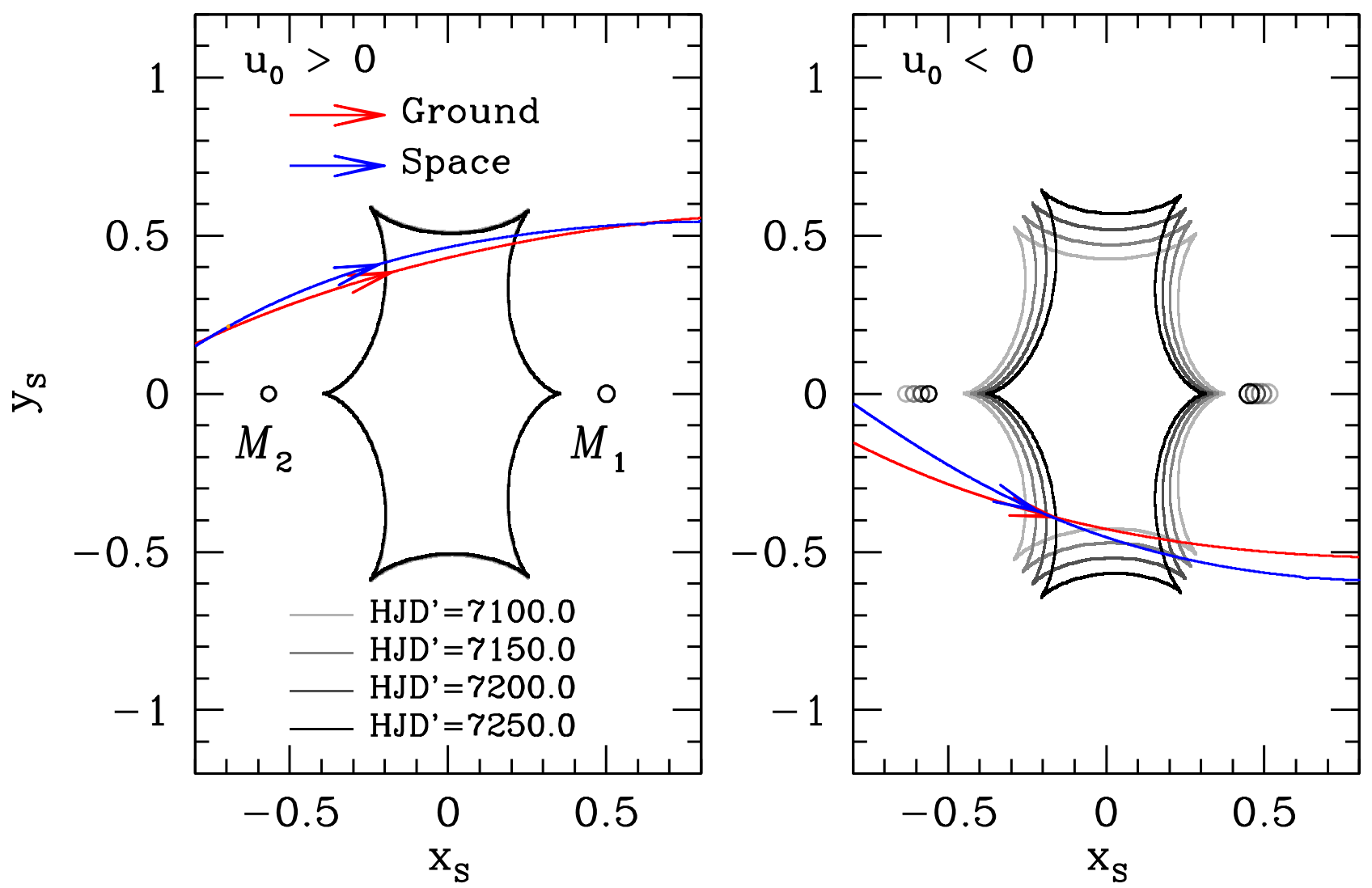

Figure 2. Geometry of the lens system for the $u_{0}>0$ (left panel) and $u_{0}<0$ (right panel) solutions. In each panel, the closed curve with six cusps represents the caustic formed by the binary lens. The locations of the binary-lens components $\left(M_{1}\right.$ and $\left.M_{2}<M_{1}\right)$ are marked by small open circles. The red and blue curves with arrows are the source trajectories as seen from Earth and from the Spitzer telescope, respectively. We note that the positions of the lens components and the shape of the caustic vary in time due to the orbital motion of the binary lens. We present the positions of the lens and caustic at four different times marked in the legend. We note that the variation of the caustic for the $u_{0}>0$ model is very small due to the small value of the orbital parameter $d s_{\perp} / d t$ and thus four different caustics appear to be a single caustic. All lengths are normalized to the angular Einstein radius corresponding to the total mass of the binary lens.
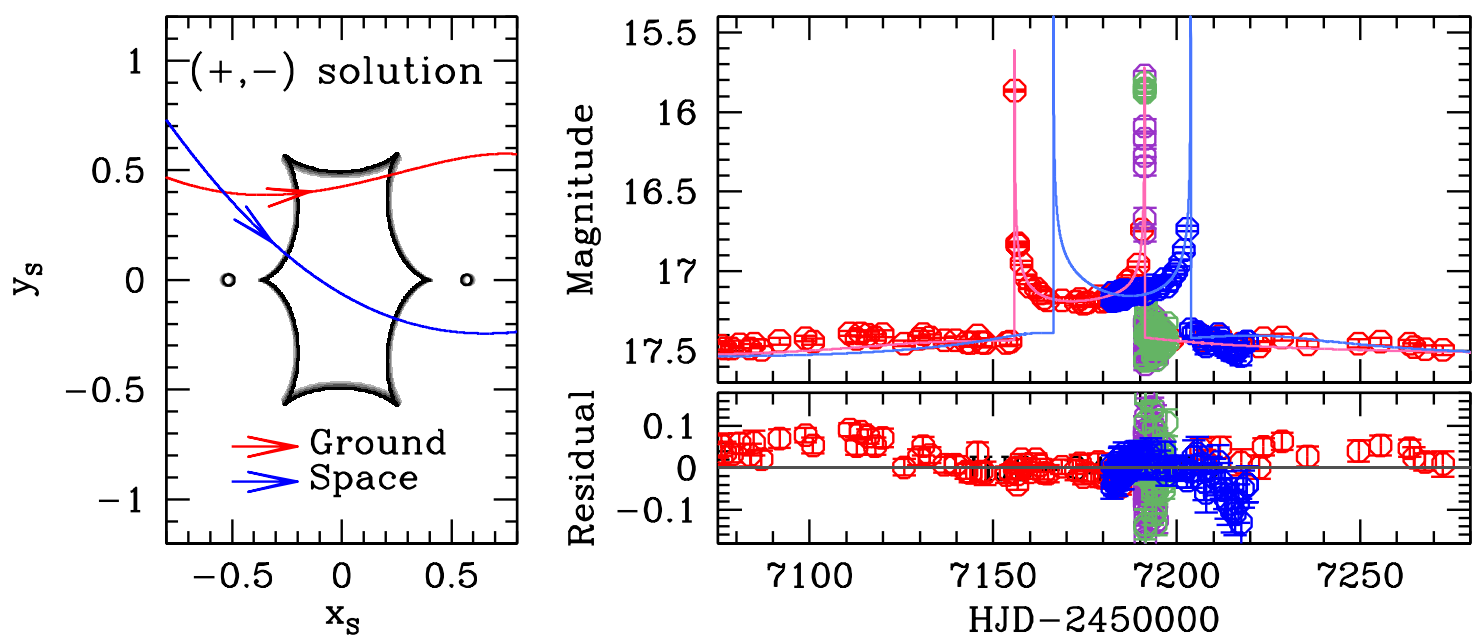

Figure 3. Lens system geometry and the light curve corresponding to the $(+,-)$ local solution. Notations are the same as those in Figures 1 and 2.

angular Einstein radius and timescale $t_{\mathrm{E}}$ by

$$
\mu_{\oplus}=\frac{\theta_{\mathrm{E}}}{t_{\mathrm{E}}} .
$$

With the additional information of $\pi_{\mathrm{E}}$, the heliocentric proper motion is determined by

$$
\boldsymbol{\mu}_{\odot}=\boldsymbol{\mu}_{\oplus}+\boldsymbol{v}_{\oplus, \perp} \frac{\pi_{\mathrm{rel}}}{\mathrm{au}},
$$

where $\boldsymbol{\mu}_{\oplus}=\mu_{\oplus}\left(\pi_{\mathrm{E}, N} / \pi_{\mathrm{E}}, \pi_{\mathrm{E}, E} / \pi_{\mathrm{E}}\right), \boldsymbol{v}_{\oplus, \perp}=(1.3,27.7) \mathrm{km} \mathrm{s}^{-1}$ is the velocity of Earth projected onto the sky at $t_{0}$, and $\pi_{\text {rel }}=\mathrm{au}\left(D_{\mathrm{L}}^{-1}-D_{\mathrm{S}}^{-1}\right)$ is the relative lens-source parallax.

\subsection{Physical Parameters}

With the space-based microlens parallax and the angular Einstein radius, the mass and distance are estimated by the relations in Equation (1). We present the determined values in 


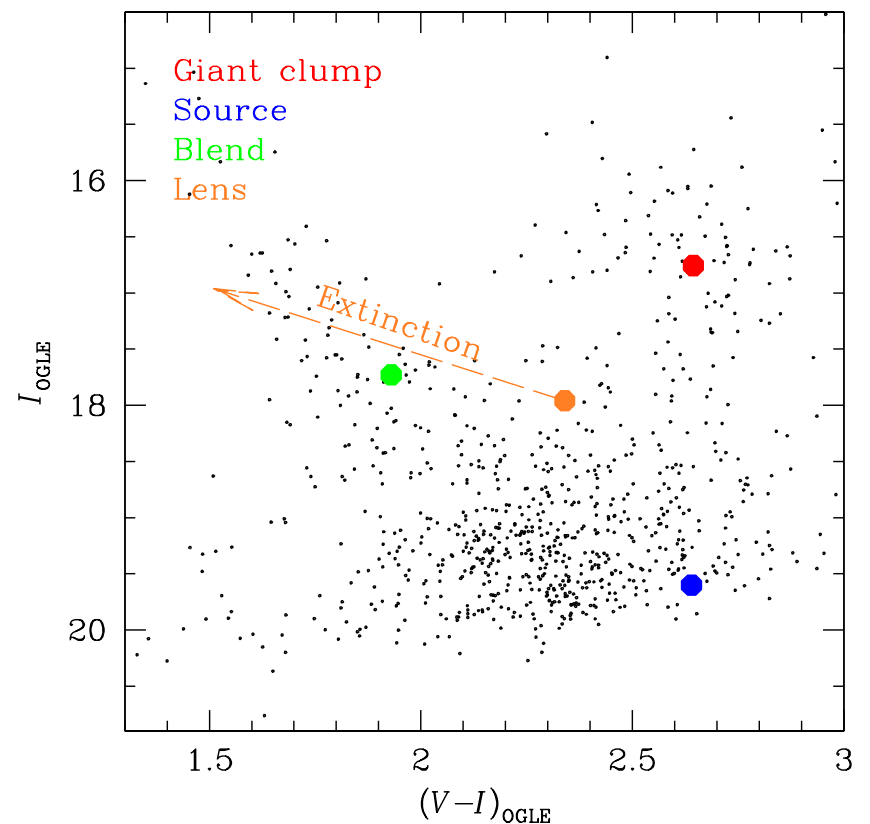

Figure 4. Position of the source star with respect to the centroid of the giant clump in the instrumental color-magnitude diagram. Also presented are the positions of the blend and the lens. The lens position is estimated under the assumption that the lens and clump giants experience the same amount of reddening and extinction. The arrow starting from the lens position represents one magnitude difference in extinction (relative to the clump), under the assumption that the ratio of total-to-selective extinction is $R_{V I}=A_{I} / E(V-I)=1.31$. Hence, if the blend is the lens, then the lens is less extincted than the clump by $\Delta A_{I} \simeq 0.5$.

Table 2

Physical Parameters

\begin{tabular}{lcc}
\hline \hline Quantity & $u_{0}>0$ & $u_{0}<0$ \\
\hline Einstein radius (mas) & $1.82 \pm 0.41$ & $1.87 \pm 0.43$ \\
Geocentric proper motion $\left(\mathrm{mas} \mathrm{yr}^{-1}\right)$ & $7.32 \pm 1.65$ & $7.90 \pm 1.82$ \\
Heliocentric proper motion $\left(\mathrm{mas} \mathrm{yr}^{-1}\right)$ & $6.16 \pm 1.39$ & $6.76 \pm 1.55$ \\
$M_{1}\left(M_{\odot}\right)$ & $1.03 \pm 0.24$ & $1.03 \pm 0.24$ \\
$M_{2}\left(M_{\odot}\right)$ & $0.91 \pm 0.21$ & $0.84 \pm 0.20$ \\
$D_{\mathrm{L}}(\mathrm{kpc})$ & $3.13 \pm 0.51$ & $2.98 \pm 0.50$ \\
$d_{\perp}(\mathrm{au})$ & $6.11 \pm 0.99$ & $6.10 \pm 1.03$ \\
$a(\mathrm{au})$ & $7.6 \pm 4.4$ & $10.8 \pm 3.6$ \\
$P($ year $)$ & $15.4 \pm 13.0$ & $23.6 \pm 8.1$ \\
Eccentricity & $0.36 \pm 0.22$ & $0.54 \pm 0.20$ \\
Inclination (degree) & $-32.9 \pm 13.3$ & $53.6 \pm 5.8$ \\
Time of perihelion (HJD') & $8158 \pm 574$ & $8032 \pm 296$ \\
\hline
\end{tabular}

Table 2 for both $u_{0}>0$ and $u_{0}<0$ solutions. We note that the two degenerate solutions have similar values of $\pi_{\mathrm{E}}$ and $\theta_{\mathrm{E}}$ and thus the estimated physical parameters are similar to each other. It is found that the binary lens responsible for OGLE-2015BLG-0479 is composed of two G-type main-sequence stars with $M_{1} \sim 1.0 M_{\odot}$ and $M_{2} \sim 0.9 M_{\odot}$ and the projected separation between the components is $d_{\perp} \sim 6$ au. The estimated distance to the lens is $D_{\mathrm{L}} \sim 3 \mathrm{kpc}$.

Since we consider a full Keplerian orbital motion, the orbital parameters are also determined. The estimated semimajor axis and orbital period are $a=7.6 \pm 4.4$ au and $P=15.4 \pm$ 13.0 years, respectively, for the $u_{0}>0$ model and $a=$ $10.8 \pm 3.6$ au and $P=23.6 \pm 8.1$ years, respectively, for the $u_{0}<0$ model. There have been numerous cases for which the projected orbital parameters $d s_{\perp} / d t$ and $d \alpha / d t$ are determined, e.g., Albrow et al. (2000). However, it is well recognized that determining the complete orbital parameters including the radial-component parameters $s_{\|}$and $d s_{\|} / d t$ is very difficult even in very favorable circumstances (Gould et al. 2013) and thus there exist only three cases for which the complete orbital parameters were measured (Shin et al. 2011, 2012; Gould et al. 2013). A major cause of the difficulty in determining the complete orbital parameters is the strong correlation between the microlens-parallax and lens-orbital effects, which have similar effects on lensing light curves. We note that the measurements of the complete orbital parameters for OGLE2015-BLG-0479 become possible because the microlens parallax is precisely measured by the Spitzer data. See Han et al. (2016) for a detailed discussion about the importance of space-based microlensing observation in characterizing orbital lens parameters.

The fact that the lens has a heavier mass than the most common lens population of low-mass stars and it is located relatively close to the observer makes us consider the possibility that the origin of blended light is likely to be the lens itself. In order to check this possibility, we mark the position of the blend in the colormagnitude diagram presented in Figure 4. We also calculate the expected position of the lens based on the lens mass (and corresponding stellar type) and lens distance, as given in Table 2. We first make this calculation under the assumption that the lens and the clump experience the same extinction (solid gold point), and then assuming that the lens suffers less extinction by an amount of $0<\Delta A_{I}<1$ (dashed gold line). The slope of the arrow, $R_{V I}=A_{I} / E(V-I)=1.3$, is determined from the ratio of total-to-selective extinction along this line of sight toward the clump. We note that the blend position is consistent with that expected for the lens provided the latter lies behind $\Delta A_{I} \simeq 0.5$ less extinction than the clump. That is, the lens would have to lie behind about three-fourths of the dust. This is quite reasonable given the lens distance of $D_{L} \simeq 3 \mathrm{kpc}$. The alternate possibility, i.e., that the blend light comes primarily from an unrelated star along the line of sight, is virtually ruled out if the microlens model is correct. This is because, regardless of how much dust lies behind the lens, its inferred I-band flux already accounts for the majority of the observed blend light. Hence, the room for other, unassociated, stars to contribute to the blend is highly restricted. With $I \sim 17.7$, the blend is bright enough for spectroscopy. Since the two components of the lens are moving with internal relative motion of the order of $\sim 15 \mathrm{~km} \mathrm{~s}^{-1}$ in both solutions, the orbit can be measured by making spectroscopic observations over a number of years.

\section{SUMMARY AND DISCUSSION}

We analyzed the combined data obtained from observations both from the ground and from the Spitzer telescope for the microlensing event OGLE-2015-BLG-0479. The light curves with strong caustic-crossing features seen from the ground and from space exhibited a time offset of $\sim 13$ days between the caustic spikes, indicating that the relative lens-source positions seen in the two places were displaced by parallax effects. From modeling the light curves, we measured the space-based microlens parallax. Combined with the angular Einstein radius measured by analyzing the caustic-crossing parts of the light curves, we determined the mass and distance of the lens. It was found that the lens was a binary composed of two G-type stars with masses of $\sim 1.0 M_{\odot}$ and $\sim 0.9 M_{\odot}$ located at a distance of 
$\sim 3 \mathrm{kpc}$. Unlike the binary event OGLE-2014-BLG-1050, which was also observed by Spitzer with similar photometric precision, cadence, and coverage, we found that interpreting OGLE-2015-BLG-0479 did not suffer from the degeneracy between $( \pm, \pm)$ and $( \pm, \mp)$ solutions, confirming that the fourfold parallax degeneracy in single-lens events collapses into the two-fold degeneracy in general binary-lens events. It was found that the location of the blend in the color-magnitude diagram was consistent with the lens properties, suggesting that the blend was the lens itself. The blend is bright enough for spectroscopy and thus the possibility can be checked from future follow-up observations.

The binary event OGLE-2015-BLG-0479 analyzed in this work demonstrates the possibility of characterizing the physical parameters of binary lenses for a significantly increased number of events. In addition to the surveys conducted in 2014 and 2015 seasons, the Spitzer microlensing survey continues in the 2016 season. In addition to Spitzer, the microlensing survey of Campaign 9 of Kepler's extended $K 2$ mission (K2C9) is being conducted in the 2016 season from which microlens parallaxes for $>127$ microlensing events are expected to be measured (Henderson et al. 2016). For these binary-lens events, the chance to measure the angular Einstein radius is high because of the greatly increased observation cadence of ground-based surveys achieved by the instrumental upgrade and the addition of new surveys, e.g., KMTNet survey (Kim et al. 2016). Being able to measure both $\pi_{\mathrm{E}}$ and $\theta_{\mathrm{E}}$, therefore, it will be possible to routinely measure the physical parameters of binary lenses.

Work by C. Han was supported by the Creative Research Initiative Program (2009-0081561) of National Research Foundation of Korea. The OGLE project has received funding from the National Science Centre, Poland, grant MAESTRO 2014/14/A/ ST9/00121 to A.U. The OGLE Team thanks Profs. M. Kubiak and G. Pietrzyński, former members of the OGLE team, for their contribution to the collection of the OGLE photometric data over the past years. Work by A.G. was supported by JPL grant 1500811. Work by J.C.Y. was performed under contract with the California Institute of Technology (Caltech)/Jet Propulsion Laboratory (JPL) funded by NASA through the Sagan Fellowship Program executed by the NASA Exoplanet Science Institute. Work by C.B.H. and Y.S. was supported by an appointment to the NASA Postdoctoral Program at the Jet Propulsion Laboratory, administered by Universities Space Research Association through a contract with NASA. The Spitzer Team thanks Christopher S. Kochanek for graciously trading us his allocated observing time on the CTIO $1.3 \mathrm{~m}$ during the Spitzer campaign. We acknowledge the high-speed internet service (KREONET) provided by Korea Institute of Science and Technology Information (KISTI).

\section{APPENDIX}

\section{ON THE ISSUE OF FALSE ALARM PROBABILITIES}

\section{A.1. Naive False Alarm Probabilities}

In principle, one can evaluate the FAPs associated with introducing either $n=2$ or $n=4$ orbital-motion parameters relative to a so-called "standard," i.e., the non-orbiting binary model,

$$
\begin{aligned}
p\left(n, \Delta \chi^{2}\right) & \equiv[\Gamma(n / 2)]^{-1} \int_{\Delta \chi^{2} / 2}^{\infty} d x x^{n / 2-1} e^{-x} \\
& \rightarrow\left[\sum_{i=0}^{n / 2} \frac{\left(\Delta \chi^{2} / 2\right)^{i}}{i !}\right] \exp \left(-\Delta \chi^{2} / 2\right),
\end{aligned}
$$

where, in the last step, we have given the explicit expression for even $n$. To evaluate these FAPs in a conservative fashion, we first renormalize that $\chi^{2}$ values in the main text so the $\chi^{2} /$ dof is exactly unity for the best model, i.e., downward by a factor of $666 / 732.9=0.909$. Then $\Delta \chi^{2}=27.3$ and $\Delta \chi^{2}=39.5$ for $n=2$ and $n=4$, respectively. The associated FAPs are then $p_{2}(27.3)=1.1 \times 10^{-6}$ and $p_{4}(39.3)=6.0 \times$ $10^{-8}$. These numbers are quite small, and one is tempted to leave it at that.

However, there is actually a deeper issue at stake, which is that it is fundamentally wrong to evaluate FAPs for this case. To understand why, we briefly recapitulate a case for which such evaluation is appropriate. This will allow us to contrast the key features of the two cases.

\section{A.2. Microlens Planet FAPs}

Suppose that a microlensing event is reasonably well fit by a point-lens model with three parameters $\left(t_{0}, u_{0}, t_{\mathrm{E}}\right)$ but is better fit by adding four additional parameters $(s, q, \alpha, \rho)$, with $q \ll 1$, indicating a planet. Without going into detail (because this is not our main focus), one can show that the FAP is approximately given by

$$
p_{\text {planet }}\left(\Delta \chi^{2}\right) \sim \frac{1}{f_{p}} \frac{2 t_{\mathrm{E}}}{t_{\mathrm{E}, p, \min }} \ln \frac{t_{\mathrm{E}}}{3 t_{\mathrm{E}, p, \min }} \exp \left(-\Delta \chi^{2} / 2\right),
$$

where $f_{p} \sim 10^{-2}$ is the fraction of all point-lens events with suitable quality data that show planetary anomalies and $t_{\mathrm{E}, p}$, $\min \sim 1 \mathrm{hr}$ is the timescale of the shortest detectable planetary anomaly. The last factor accounts for the $\chi^{2}$ distribution associated with two additional parameters $(s, \rho)$, while the first three count the effective number of trials. The first quantifies how many events are searched for each real planet. The second quantifies the number of independent locations along the light curve (effectively parameterized by $\alpha$ ) at which one can search for planets. The third counts the number of independent durations of this perturbation at a fixed location (effectively parameterized by $q$ ). For typical Einstein timescales $t_{\mathrm{E}} \sim 30$ days, the first three factors combine to a value of $\sim 10^{6}$.

Now, such FAPs are never calculated in practice for the simple reason that no one has ever considered a microlensing planet to be "detectable" unless $\Delta \chi^{2}>160$ (Gaudi et al. 2002), and in fact all reported detections have had substantially higher $\Delta \chi^{2}$. Even at the putative threshold of detection; however, the FAP is $\sim 10^{-29}$. The reasons for this conservative attitude do not concern us here, but the interested reader can consult Gaudi et al. (2002) and Yee et al. (2012, 2013).

Our focus is rather on a matter of principle. A planet with a mass ratio of $q=0$ yields an absolutely identical model light curve as a point lens. Hence, if we "measure" a mass ratio $q=(1.0 \pm 1.0) \times 10^{-4}$, we do not say that we have "detected a planet, possibly of zero mass." Rather, we formulate this as an upper limit on the mass of any possible planet that is present (at a given $(s, \alpha)$ ). On the other hand, if the "measurement" 
were $q=(1.0 \pm 0.2) \times 10^{-4}$, then we would think naively that we may have detected a planet and might ask questions about the FAP. (As mentioned in the previous paragraph, no such "detection" would ever be considered, but if it were, then inserting $\Delta \chi^{2}=25$, one finds that the FAP would exceed unity!)

\section{A.3. Orbiting Binary FAPs}

OGLE-2015-BLG-0479 shows two clear caustic crossings, and there are no known astrophysical phenomena that can generate such light-curve features except having two masses projected on the sky within of order one Einstein radius of each other. These two masses must either be bound to each other (and so in a Kepler orbit) or are unbound, i.e., merely seen in projection, in which case they are moving relative to each other in rectilinear, unaccelerated motion. (The probability of the latter is quite low, as we discuss immediately below). In either case, one knows a priori that they have some instantaneous relative motion. Hence, one is not "adding parameters" to include such motion $(d s / d t$ and $d \alpha / d t$ in the formulations in the main text). Rather, the opposite is true: if one were to model this-or any-binary system (bound or unbound) without including transverse relative velocity parameters, one would be suppressing the impact of known physics on the light curve and so, possibly, introducing systematic errors on the remaining parameters being measured.

In particular, if this measurement showed a best fit of zero relative motion (or consistent with zero motion at low $\Delta \chi^{2}$ ), we would not say (as with $q \simeq 0$ in the planet case) that we had failed to measure transverse motion. Still less would we say that there was "no justification" for introducing transversevelocity parameters. Rather, we would say that we had measured the transverse velocity to be close to zero, and this measurement would be quite important because it would provide additional evidence that the system is bound.

That is, the probability of finding two unrelated stars projected within about one Einstein radius is already low, but the probability that they are moving slowly with respect to each other is yet another factor of $\sim 100$ lower. The first probability is roughly the optical depth to microlensing, i.e., $\tau \sim 10^{-6}$. This might seem too small to consider, but since there have been more than 20,000 microlensing events discovered to date, the probability of such a chance projection in one of these is a few percent. Hence, the additional suppression factor of $~ 100$ from measuring a small transverse motion can be important.

Once the binary is demonstrated to be bound with very high probability, it is certainly justified to "introduce" the remaining two parameters needed to describe a full Kepler orbit. We have put "introduce" in quotes because nothing is being introduced: rather we are simply not eliminating parameters that are known to be required to describe the physical system.

\section{A.4. When Is One Justified in Eliminating Some or All Kepler Parameters?}

From a purist standpoint, the answer is never. The practical reason that these "extra" parameters are frequently excluded is that in many cases nothing would be measured by doing so. For example, in many cases, one finds that the transverse motion is consistent with zero but is equally consistent with values several times higher than permitted for bound orbits. Since, as just mentioned, the prior probability for bound orbits is quite high, such a "measurement" yields no information. One is then tempted to simply set this motion to zero, i.e., fit the data without these two parameters. For many years, this is exactly what was done. However, such an approach is unphysical: binary stars do not "stand still." Furthermore, as first shown by Batista et al. (2011) and then further elaborated on by Skowron et al. (2011), if the transverse motion parameters are arbitrarily set to zero, then this can introduce systematic errors into the parallax parameters, with which they are correlated. Rather, the correct approach is to maintain these parameters. Then if they take on improbable or unphysical values, the proper way to handle this is to introduce Bayesian priors on these parameters. See, for example, Poleski et al. (2014). On the other hand, if the light curve does not contain enough information to fruitfully constrain either the transverse motion parameters or the parallax parameters, then setting these to zero is in most cases an appropriate way to simplify the fitting, since the remaining parameters are usually not strongly correlated with them.

\section{REFERENCES}

Alard, C., \& Lupton, R. H. 1998, ApJ, 503, 325

Albrow, M. D., Beaulieu, J.-P., Caldwell, J. A. R., et al. 2000, ApJ, 534, 894

Batista, V., Gould, A., Dieters, S., et al. 2011, A\&A, 529, 110

Bensby, T., Adén, D., Meléndez, J., et al. 2011, A\&A, 533, A134

Bessell, M. S., \& Brett, J. M. 1988, PASP, 100, 1134

Bramich, D. M. 2008, MNRAS, 386, L77

Calchi Novati, S., Gould, A., Udalski, A., et al. 2015a, ApJ, 804, 20

Calchi Novati, S., Gould, A., Yee, J. C., et al. 2015b, ApJ, 814, 92

Claret, A. 2000, A\&A, 363, 1081

Gaudi, B. S. 2012, ARA\&A, 50, 411

Gaudi, B. S., Albrow, M. D., \& An, J. 2002, ApJ, 566, 463

Gould, A. 1994, ApJL, 421, L75

Gould, A. 2008, ApJ, 681, 1593

Gould, A., Carey, S., \& Yee, J. 2014, sptz prop, 11006

Gould, A., Shin, I.-G., Han, C., et al. 2013, ApJ, 768, 126

Gould, A., Udalski, A., An, D., et al. 2006, ApJL, 644, L37

Han, C., Udalski, A., Gould, A., et al. 2016, ApJ, 827, 11

Henderson, C. B., Poleski, R., Penny, M., et al. 2016, PASP, submitted

Kervella, P., Bersier, D., Mourard, D., et al. 2004, A\&A, 428, 587

Kim, S.-L., Lee, C.-U., Park, B.-G., et al. 2016, JKAS, 49, 37

Nataf, D. H., Gould, A., Fouqué, P., et al. 2013, ApJ, 769, 88

Park, H., Udalski, A., Han, C., et al. 2013, ApJ, 778, 134

Pejcha, O., \& Heyrovský, D. 2009, ApJ, 690, 1772

Poleski, R., Udalski, A., Dong, S., et al. 2014, ApJ, 782, 47

Refsdal, S. 1966, MNRAS, 134, 315

Schneider, P., \& Weiss, A. 1986, A\&A, 164, 237

Shin, I.-G., Han, C., Choi, J.-Y., et al. 2012, ApJ, 755, 91

Shin, I.-G., Udalski, A., Han, C., et al. 2011, ApJ, 735, 85

Shvartzvald, Y., Udalski, A., Gould, A., et al. 2015, ApJ, 814, 111

Skowron, J., Udalski, A., Gould, A., et al. 2011, ApJ, 738, 87

Street, R. A., Udalski, A., Calchi Novati, A., et al. 2016, ApJ, 819, 93

Udalski, A. 2003, AcA, 53, 291

Udalski, A., Szymanski, M. K., \& Szymanksi, G. 2015a, AcA, 65, 1

Udalski, A., Yee, J. C., Gould, A., et al. 2015b, ApJ, 799, 237

Woźniak, P. R. 2000, AcA, 50, 421

Yee, J. C., Gould, A., Beichman, C., et al. 2015, ApJ, 810, 155

Yee, J. C., Hung, L.-W., Bond, I. A., et al. 2013, ApJ, 769, 77

Yee, J. C., Svartzvald, Y., Gal-Yam, A., et al. 2012, ApJ, 755, 102

Yoo, J., DePoy, D. L., Gal-Yam, A., et al. 2004, ApJ, 603, 139

Zhu, W., Udalski, A., Gould, A., et al. 2015, ApJ, 805, 8 